\title{
Principal Component Analysis of Physiological Traits Governing Drought Tolerance in Germplasm Accessions of Green Gram [Vigna radiata (L.)]
}

\author{
M. S. P. Kanavi ${ }^{*}$, N. Nagesha ${ }^{2}$, G. Somu ${ }^{3}$, B. T. Krishnaprasad ${ }^{4}$ and S. Rangaiah ${ }^{5}$ \\ ${ }^{1}$ Department of Genetics and Plant Breeding, College of Agriculture, Hassan, \\ University of Agricultural Sciences, Bangalore, Karnataka, India \\ ${ }^{2}$ Department of Plant Biotechnology, College of Agriculture, G.K.V.K, \\ University of Agricultural Sciences, Bangalore, Karnataka, India \\ ${ }^{3}$ Assistant Breeder, AICRP on Sorghum, Chamarajanagara, \\ University of Agricultural Sciences, Bangalore, Karnataka, India \\ ${ }^{4}$ Department of Agricultural Biotechnology, College of Agriculture, Hassan, \\ University of Agricultural Sciences, Bangalore, Karnataka, India \\ ${ }^{5}$ Department of Genetics and Plant Breeding, College of Agriculture, G.K.V.K, \\ University of Agricultural Sciences, Bangalore, Karnataka, India \\ *Corresponding author
}

\section{A B S T R A C T}

Keywords

Green gram,

Drought tolerance,

Physiological

traits,Principal

component analysis,

Biplot analysis

Article Info

Accepted:

25 February 2020

Available Online:

10 March 2020
An experiment was conducted to evaluate 200 green gram germplasm accessions along with five check entries for drought tolerance using augmented design during summer 2015 by imposing drought stress condition. Observations were recorded on 17 quantitative traits. ANOVA revealed high significant differences among germplasm accessions for yield and for drought tolerant physiological traits. Mean squares attributable to 'Genotypes vs check entries' were significant for all the traits except relative water content. Principal component analysis was carried out for 6physiological traits showing positive correlation with yield. Out of 6 factors generated by PCA, there are only 2 factors with eigenvalues more than or close to one contributing for more than $88.03 \%$ of variability. Among the variables studied, variable proline content (11.91) had highest per cent contribution to the total variability followed by spad chlorophyll meter reading (10.80), leaf water potential (10.75), Relative water content (8.51) and specific leaf area (6.31).

\section{Introduction}

Green gram [Vigna radiata (L.) Wilczek] also known as mung bean is an important short duration pulse crop of the tropical and subtropical countries of the world. . It is a prehistoric crop and grown throughout Asia. Green gram is the third most important pulse crop of India after chickpea and red gram.It belongs to papilionoid subfamily of the 
Fabaceae family and has a diploid chromosome number of $2 n=2 x=22$. Green gram as a legume crop has the ability to fix atmospheric nitrogen via root rhizobial symbiosis leading to improved soil fertility and texture (Graham and Vance, 2003).The protein content of pulses are twice that of cereals (20-25\%) and almost equal to that of meat and poultry hence commonly pulses are called as poor man's meat (Reddy, 2009).India is the major pulse producing country in the world which shares $30-35 \%$ and $27-28 \%$ of the total area and production respectively (Shao et al., 2018). In India mung bean is cultivated in an area of 40.70 lakh hectare with production of 19.01 lakh tones and productivity of $467 \mathrm{~kg} \mathrm{ha}^{-1}$.

Average productivity of mung bean in India is one of the lowest compared to world average since it is mainly cultivated on marginal and poor fertile soils under rainfed condition in rabi or late rabi season utilizing available residual soil moisture after harvesting kharif crop. Hence crop is expected to undergo several kinds of droughts during its cropping period.

Crop is likely to experience severe droughts in days to come because of global warming and climate change which are adding to the woes of reduced soil moisture availability to crop growth and production. Drought is the major environmental stress severely impairing plant growth and development limiting performance and production of crop plants than any other environmental stresses (Shao et al., 2009). Drought is the major constraint for green gram production in India due to erratic and insufficient rainfall (Baroowa and Gogoi., 2015).

Droughtis a multidimensional stress which disturbs normal metabolism and yield of crop plants. Climate change at global level is rapidly increasing the frequency of severe drought conditions (Dai, 2012).
The plants possess a wide range of physiological and genetic adaptations innate or triggered to combat the stress ranging from transient responses to low soil moisture to major survival mechanisms of escape by early flowering in absence of seasonal rainfall (Supratima, 2016).Some of the commonly observed drought tolerance mechanisms adapted by the plants are; longer root length, longer root to shoot ratio, leaf waxing, reduced transpiration, reduced photosynthesis, proline accumulation, ABA accumulation, prevention of chlorophyll degradation, ionic balance, balance of water status, stomatal behavior, photosynthetic efficiency, carbon allocation and utilization. Studying water stressthrough quantification of physiological responses of plants under water stress is a viable, reliable and accurate approach (Kursar et al., 2009).Selection efficiency in breeding for water stresscould be enhanced if particular physiological or morphological attributes related to yield under stress environment could be identified and employed as selection criteria for complementing traditional plant breeding (Rowland et al., 2018).While designing a breeding program to improve drought tolerance of a crop plant, it is necessary to gain knowledge concerning both the genetics and physiological mechanisms (Clarke and Townley, 1984). Therefore, physiological traits with strong correlation of plants response to drought are crucial in understanding and exploring water stress mechanisms (Maréchaux et al., 2015).

Multivariate analysis such as principal component analysis usually starts out with data involving a substantial number of correlated variables. Principal Component Analysis (PCA) is a very powerful dimension-reduction technique that can be used to reduce a large set of variables to a small set of variables that still contains most of the information of the larger set. Principal component analysis(PCA) is a statistical / 
mathematical procedure that transforms more number of correlated variables into a smaller number of uncorrelated variables called as principal components.

The first principal component with highest PCA coefficient /eigenvalue accounts for as much of the variability in the data as possible, and each succeeding component accounts for as much of the remaining variability as possible with corresponding PCA coefficients /eigenvalues.

\section{Materials and Methods}

The experiment was conducted at experimental plot of College of Agriculture, Hassan, University of Agricultural Sciences, Bangalore. The experimental site is geographically located at Southern Transitional Zone (Zone-7) of Karnataka with an altitude of $827 \mathrm{~m}$ above Mean Sea Level (MSL) and at $33^{\prime} \mathrm{N}$ latitude and $75^{\circ} 33^{\prime}$ to $76^{\circ}$ E38' longitude. The study material consisted of 200 germplasm accessions collected from different research institutions / organizations representing different agro-climatic zones. List of germplasm accessions used in the study with their source is given in table No1.

\section{Layout of the experiment}

The experiment was conducted in an Augmented Randomized Complete Block Design with 200germplasm accessions. As per the augmented RCBD, the check entries were replicated twice randomly in each block. There were 5 blocks, each block had 5 plots of size $3 \times 3 \mathrm{~m}^{2}$ thus each block size was 15 $\mathrm{m}^{2}$. The gross area of experimental plot was $75 \mathrm{~m}^{2}$. The row spacing was $30 \mathrm{~cm}$ and inter plant distance was $10 \mathrm{~cm}$. The experiment was conducted during summer 2015. Recommended crop production practices were followed to raise healthy crop.

\section{Imposing drought condition}

Drought condition was imposed by withholding irrigation 25 days after sowing (Baroowa and Gogoi, 2015; Pooja et al., 2019). Since the experiment was conducted during summer season, there were no unpredicted rains during the entire cropping period hence the drought condition was effectively imposed. The rainfall data of experimental site during the cropping period is given in table No.2.

\section{Plant sampling and data collection}

Observations were recorded on five randomly chosen competitive plants from each germplasm accession for all the physiological traits. The values of five competitive plants were averaged and expressed as mean of the respective characters. The observations were taken on the traits like; Threshing \%, Harvest index (\%),SCMR (SPAD Chlorophyll meter reading), Leaf water potential(Mpa), Proline content $\left(\mu \mathrm{gg}^{-1}\right)$, Relative water content, Specific leaf area and Seed yield per plant.

\section{Statistical analysis}

\section{Analysis of variance (ANOVA)}

The quantitative trait mean value of five randomly selected plants in each of the genotype and check entries were used for statistical analysis. ANOVA was performed to partition the total variation among genotypes and check entries into sources attributable to 'Genotypes + Check entries', Genotypes', Check entries' and Genotypes vs check entries', following the augmented design as suggested by Federer (1956) using statistical package for augmented design SAS version 9.3 and IndoStat. The adjusted trait mean of each of the genotype was estimated (Federer, 1956) and the same was used for all subsequent statistical analysis. 


\section{Multivariate analysis}

Factor analysis, using the Principal Component Analysis (PCA) as extraction method and Varimax rotation, was performed to verify if the assay data variation and obtained factors could explain genotype performance and identify drought tolerance controlling factors.

Biplot analysis was presented by first two principal component analysis (PCA) which were computed based on rank correlation matrix using data from 6 physiological traits by Microsoft Excel (2007) and XLSTAT 2014, Copyright Addinsoft 1995-2014 (http://www.xlstat.com) as described by Iqbal et al., (2014).

\section{Results and Discussion}

\section{Analysis of variance (ANOVA)}

Analysis of variance revealed highly significant mean squares attributable to germplasm accessions for all the traits. Significant mean squares were recorded for all the traits. (Table 3). Mean squares attributable to 'Genotypes $v s$ check entries' were significant for all the traits except relative water content. These results suggest significant differences among the germplasm accessions. The germplasm accessions as group differed significantly for all of the traits under investigation, similarly, check entries as group differed significantly for most of the traits under study.Hemavathy et al., (2014) has also reported that green gram germplasm differed significantly for all the traits under study.

\section{Multivariate analysis}

The first principal component with highest PCA coefficient/ eigenvalue accounts for as much of the variability in the data as possible, and each succeeding component accounts for as much of the remaining variability as possible with corresponding eigenvalue /PCA coefficient.

Principal component analysis has to be performed only for those traits (independent variables) having positive correlation with dependent variable yield. Hence correlation studies were first carried out to identify physiological traits to be considered for principal component analysis.

\section{Correlation coefficient analysis}

Correlation coefficients are used to measure the strength of the relationship between two variables (dependent and independent). Pearson correlation which is one of the most commonly used statistics was performed. Among the independent variables, proline content had highest positive correlation with seed yield per plant $(0.63)$ followed by spad chlorophyll meter reading (0.62), leaf water potential (0.61), harvest index (0.60), relative water content $(0.51)$ and specific leaf area (0.41). Correlation matrix is given in the table 4.

Sandhiya and Saravanan (2018) have reported significant positive correlation with traits, number of pods per plant, number of clusters per plantand number of pods per cluster in mungbean.Pooja et al., (2019) has also reported positive correlation of leaf area with RWC (0.57), membrane stability index (0.39) and protein content $(0.35)$ under drought condition in green gram.

\section{Kaiser-Meyer-Olkin (KMO) test}

KMO test measures whether the data is suitable for factor analysis like PCA. The test measures sampling adequacy for each variable in the model andfor complete model. Lower the proportion, more the data is suited 
for factor analysis. KMO values between 0.8 and 1 indicate the sampling is adequate. KMO values less than 0.6 indicate the sampling is not adequate and that remedial action should be taken. KMO test results are given in table 5.All the 6 physiological traits considered for study satisfied the conditions of KMO test

\section{Principal component analysis}

Principal component analysis of physiological traits governing drought tolerance was performed and eigenvalues are presented in table 6. The first two factors explain 88.03 per cent of the total variability controlled by physiological traits. Highest factor loadings / component coefficients were recorded by proline content (0.98) followed by Spad chlorophyll meter reading, leaf water potential (0.92), relative water content (0.87), harvest index (0.78) and specific leaf area $(0.72)$. Factor loading values are presented in table 7.

The analysis will simply identify factors / principal component numbers. It is the researcher who has to decide which variable to be considered. In making decision to identify variables depending upon our research interest one should refer to factor loadings or component coefficient values which are correlation coefficients between variables and the factors. These values will help in making decision to identify variables having maximum contribution for total variability

Table.1 List of germplasm accessions used in the study and their source

\begin{tabular}{|l|l|l|}
\hline SI. No. & Germplasm & Location \\
\hline $\mathbf{1}$ & KM13-16 & ARS, Bidar \\
\hline $\mathbf{2}$ & KM13-19 & ARS, Bidar \\
\hline $\mathbf{3}$ & KM13-39 & ARS, Bidar \\
\hline $\mathbf{4}$ & GG13-7 & ARS, Bidar \\
\hline $\mathbf{5}$ & GG13-6 & ARS, Bidar \\
\hline $\mathbf{6}$ & KM13-44 & ARS, Bidar \\
\hline $\mathbf{7}$ & GG13-10 & ARS, Bidar \\
\hline $\mathbf{8}$ & SML-668 & ARS, Bidar \\
\hline $\mathbf{9}$ & KM13-9 & ARS, Bidar \\
\hline $\mathbf{1 0}$ & IPM99-125 & ARS, Bidar \\
\hline $\mathbf{1 1}$ & LGG-596 & RARS, Guntur \\
\hline $\mathbf{1 2}$ & LGG-572 & RARS, Guntur \\
\hline $\mathbf{1 3}$ & LGG-450 & RARS, Guntur \\
\hline $\mathbf{1 4}$ & LGG-583 & RARS, Guntur \\
\hline $\mathbf{1 5}$ & LGG-590 & RARS, Guntur \\
\hline $\mathbf{1 6}$ & LGG-588 & RARS, Guntur \\
\hline $\mathbf{1 7}$ & LGG-589 & RARS, Guntur \\
\hline $\mathbf{1 8}$ & LGG-579 & RARS, Guntur \\
\hline $\mathbf{1 9}$ & LGG-562 & RARS, Guntur \\
\hline $\mathbf{2 0}$ & LGG-582 & RARS, Guntur \\
\hline $\mathbf{2 1}$ & LGG-585 & RARS, Guntur \\
\hline $\mathbf{2 2}$ & AKL-170 & NBPGR, Akola \\
\hline $\mathbf{2 3}$ & PLM-110 & UAS, Bangalore \\
\hline & & \\
\hline
\end{tabular}




\begin{tabular}{|c|c|c|}
\hline 24 & LGG-577 & RARS, Guntur \\
\hline 25 & IC-436624 & IIPR, Kanpur \\
\hline 26 & IC-436723 & IIPR, Kanpur \\
\hline 27 & IC-413316 & IIPR, Kanpur \\
\hline 28 & IC-436746 & IIPR, Kanpur \\
\hline 29 & VGG10-010 & TNAU, Coimbatore \\
\hline 30 & VGG04-011 & TNAU, Coimbatore \\
\hline 31 & VGG04-007 & TNAU, Coimbatore \\
\hline 32 & COGG-93 & TNAU, Coimbatore \\
\hline 33 & VBNGG-2 & TNAU, Coimbatore \\
\hline 34 & $\begin{array}{l}\text { TARM- } \\
2013\end{array}$ & TNAU, Coimbatore \\
\hline 35 & VGG04-005 & TNAU, Coimbatore \\
\hline 36 & COGG-920 & TNAU, Coimbatore \\
\hline 37 & VGG07-003 & TNAU, Coimbatore \\
\hline 38 & VGG10-002 & TNAU, Coimbatore \\
\hline 39 & VGG-112 & TNAU, Coimbatore \\
\hline 40 & IC-92048 & NBPGR, Akola \\
\hline 41 & AKL-103 & NBPGR, Akola \\
\hline 42 & AKL-39 & NBPGR, Akola \\
\hline 43 & AKL-106 & NBPGR, Akola \\
\hline 44 & AKL-225 & NBPGR, Akola \\
\hline 45 & AKL-95 & NBPGR, Akola \\
\hline 46 & AKL-194 & NBPGR, Akola \\
\hline 47 & AKL-212 & NBPGR, Akola \\
\hline 48 & AKL-195 & NBPGR, Akola \\
\hline 49 & AKL-211 & NBPGR, Akola \\
\hline 50 & KM13-11 & ARS, Bidar \\
\hline 51 & KM13-30 & ARS, Bidar \\
\hline 52 & KM13-45 & ARS, Bidar \\
\hline 53 & KM13-18 & ARS, Bidar \\
\hline 54 & KM13-5 & ARS, Bidar \\
\hline 55 & KM13-02 & ARS, Bidar \\
\hline 56 & KM13-37 & ARS, Bidar \\
\hline 57 & KM13-23 & ARS, Bidar \\
\hline 58 & KM13-55 & ARS, Bidar \\
\hline 59 & KM13-12 & ARS, Bidar \\
\hline 60 & GG13-9 & ARS, Bidar \\
\hline 61 & KM13-49 & ARS, Bidar \\
\hline 62 & GG13-4 & ARS, Bidar \\
\hline 63 & GG13-54 & ARS, Bidar \\
\hline 64 & KM13-20 & ARS, Bidar \\
\hline 65 & GG13-5 & ARS, Bidar \\
\hline
\end{tabular}




\begin{tabular}{|c|c|c|}
\hline 66 & Chinamung & ARS, Bidar \\
\hline 67 & GG13-2 & ARS, Bidar \\
\hline 68 & KM13-26 & ARS, Bidar \\
\hline 69 & KM13-47 & ARS, Bidar \\
\hline 70 & KM13-41 & ARS, Bidar \\
\hline 71 & KM13-11 & ARS, Bidar \\
\hline 72 & KM13-42 & ARS, Bidar \\
\hline 73 & GG13-11 & ARS, Bidar \\
\hline 74 & GG13-8 & ARS, Bidar \\
\hline 75 & GG13-12 & ARS, Bidar \\
\hline 76 & KM13-48 & ARS, Bidar \\
\hline 77 & IPM2-3 & ARS, Bidar \\
\hline 78 & IPM2-14 & ARS, Bidar \\
\hline 79 & PDM-139 & ARS, Bidar \\
\hline 80 & LGG-580 & RARS, Guntur \\
\hline 81 & PM-112 & TNAU, Coimbatore \\
\hline 82 & LGG-578 & NBPGR, Akola \\
\hline 83 & LGG-563 & NBPGR, Akola \\
\hline 84 & LGG-594 & NBPGR, Akola \\
\hline 85 & TM-96-2 & NBPGR, Akola \\
\hline 86 & LGG-593 & NBPGR, Akola \\
\hline 87 & LGG-591 & NBPGR, Akola \\
\hline 88 & PM-115 & NBPGR, Akola \\
\hline 89 & LGG-587 & NBPGR, Akola \\
\hline 90 & PM-113 & NBPGR, Akola \\
\hline 91 & LGG-586 & NBPGR, Akola \\
\hline 92 & IC-436775 & NBPGR, Akola \\
\hline 93 & IC-413311 & NBPGR, Akola \\
\hline 94 & IC-398984 & NBPGR, Akola \\
\hline 95 & IC-436767 & NBPGR, Akola \\
\hline 96 & IC-436573 & NBPGR, Akola \\
\hline 97 & LGG-584 & NBPGR, Akola \\
\hline 98 & LGG-592 & NBPGR, Akola \\
\hline 99 & LGG-555 & NBPGR, Akola \\
\hline 100 & LGG-564 & NBPGR, Akola \\
\hline 101 & LGG-460 & RARS, Guntur \\
\hline 102 & LGG-595 & RARS, Guntur \\
\hline 103 & LGG-566 & RARS, Guntur \\
\hline 104 & IC-553514 & IIPR, Kanpur \\
\hline 105 & IC-413319 & IIPR, Kanpur \\
\hline 106 & IC-436542 & IIPR, Kanpur \\
\hline 107 & IC-546493 & IIPR, Kanpur \\
\hline 108 & IC-436594 & IIPR, Kanpur \\
\hline
\end{tabular}




\begin{tabular}{|c|c|c|}
\hline 109 & IC-436630 & IIPR, Kanpur \\
\hline 110 & IC-436668 & IIPR, Kanpur \\
\hline 111 & IC-436555 & IIPR, Kanpur \\
\hline 112 & IC-413314 & IIPR, Kanpur \\
\hline 113 & AKL-20 & NBPGR, Akola \\
\hline 114 & AKL-89 & NBPGR, Akola \\
\hline 115 & AKL-228 & NBPGR, Akola \\
\hline 116 & AKL-184 & NBPGR, Akola \\
\hline 117 & AKL-182 & NBPGR, Akola \\
\hline 118 & AKL-230 & NBPGR, Akola \\
\hline 119 & AKL-229 & NBPGR, Akola \\
\hline 120 & AKL-86 & NBPGR, Akola \\
\hline 121 & IC-436646 & IIPR, Kanpur \\
\hline 122 & IC-343964 & IIPR, Kanpur \\
\hline 123 & IC-436528 & IIPR, Kanpur \\
\hline 124 & IC-436723 & IIPR, Kanpur \\
\hline 125 & IC-546491 & IIPR, Kanpur \\
\hline 126 & IC-546481 & IIPR, Kanpur \\
\hline 127 & IC-398988 & IIPR, Kanpur \\
\hline 128 & VGG10-005 & TNAU, Coimbatore \\
\hline 129 & VBN-223 & TNAU, Coimbatore \\
\hline 130 & COGG-912 & TNAU, Coimbatore \\
\hline 131 & VBN(G9)-3 & TNAU, Coimbatore \\
\hline 132 & ML-1165 & TNAU, Coimbatore \\
\hline 133 & VGG04-025 & TNAU, Coimbatore \\
\hline 134 & VGG04-004 & TNAU, Coimbatore \\
\hline 135 & VGG04-149 & TNAU, Coimbatore \\
\hline 136 & COGG-954 & TNAU, Coimbatore \\
\hline 137 & VGG08-002 & TNAU, Coimbatore \\
\hline 138 & VBN-1 & TNAU, Coimbatore \\
\hline 139 & VGG-119 & TNAU, Coimbatore \\
\hline 140 & VC3890-A & TNAU, Coimbatore \\
\hline 141 & DGGV-4 & UAS, Raichur \\
\hline 142 & KPS-1 & UAS, Raichur \\
\hline 143 & CGG-973 & UAS, Raichur \\
\hline 144 & CN9-5 & UAS, Raichur \\
\hline 145 & KPS-2 & UAS, Raichur \\
\hline 146 & VC-6173 & UAS, Raichur \\
\hline 147 & VC-6368 & UAS, Raichur \\
\hline 148 & $\mathrm{CO}-6$ & UAS, Raichur \\
\hline 149 & Harsha & UAS, Raichur \\
\hline 150 & PLM-92 & UAS, Bangalore \\
\hline 151 & MH-709 & UAS, Raichur \\
\hline
\end{tabular}




\begin{tabular}{|c|c|c|}
\hline 152 & LGG-460 & RARS, Guntur \\
\hline 153 & KGS-5 & UAS, Raichur \\
\hline 154 & Barimung-4 & UAS, Raichur \\
\hline 155 & AKL-189 & NBPGR, Akola \\
\hline 156 & AKL-168 & NBPGR, Akola \\
\hline 157 & AKL-218 & NBPGR, Akola \\
\hline 158 & AKL-179 & NBPGR, Akola \\
\hline 159 & AKL-185 & NBPGR, Akola \\
\hline 160 & AKL-163 & NBPGR, Akola \\
\hline 161 & COGG-912 & TNAU, Coimbatore \\
\hline 162 & IC-73451 & NBPGR, Akola \\
\hline 163 & IC-105690 & NBPGR, Akola \\
\hline 164 & IC-73534 & NBPGR, Akola \\
\hline 165 & IC-73412 & NBPGR, Akola \\
\hline 166 & IC-39605 & NBPGR, Akola \\
\hline 167 & IC-73472 & NBPGR, Akola \\
\hline 168 & IC-92053 & NBPGR, Akola \\
\hline 169 & IC-73779 & NBPGR, Akola \\
\hline 170 & IC-73462 & NBPGR, Akola \\
\hline 171 & IC-118992 & NBPGR, Akola \\
\hline 172 & IC-53783 & NBPGR, Akola \\
\hline 173 & IC-73456 & NBPGR, Akola \\
\hline 174 & IC-73458 & NBPGR, Akola \\
\hline 175 & AKL-105 & NBPGR, Akola \\
\hline 176 & AKL-213 & NBPGR, Akola \\
\hline 177 & AKL-169 & NBPGR, Akola \\
\hline 178 & AKL-220 & NBPGR, Akola \\
\hline 179 & AKL-84 & NBPGR, Akola \\
\hline 180 & AKL-82 & NBPGR, Akola \\
\hline 181 & AKL-97 & NBPGR, Akola \\
\hline 182 & AKL-226 & NBPGR, Akola \\
\hline 183 & AKL-24 & NBPGR, Akola \\
\hline 170 & IC-73462 & NBPGR, Akola \\
\hline 171 & IC-118992 & NBPGR, Akola \\
\hline 172 & IC-53783 & NBPGR, Akola \\
\hline 173 & IC-73456 & NBPGR, Akola \\
\hline 174 & IC-73458 & NBPGR, Akola \\
\hline 175 & AKL-105 & NBPGR, Akola \\
\hline 176 & AKL-213 & NBPGR, Akola \\
\hline 177 & AKL-169 & NBPGR, Akola \\
\hline 178 & AKL-220 & NBPGR, Akola \\
\hline 179 & AKL-84 & NBPGR, Akola \\
\hline 180 & AKL-82 & NBPGR, Akola \\
\hline
\end{tabular}




\begin{tabular}{|l|l|l|}
\hline $\mathbf{1 8 1}$ & AKL-97 & NBPGR, Akola \\
\hline $\mathbf{1 8 2}$ & AKL-226 & NBPGR, Akola \\
\hline $\mathbf{1 8 3}$ & AKL-24 & NBPGR, Akola \\
\hline $\mathbf{1 8 4}$ & AKL-174 & NBPGR, Akola \\
\hline $\mathbf{1 8 5}$ & AKL-161 & NBPGR, Akola \\
\hline $\mathbf{1 8 6}$ & AKL-180 & NBPGR, Akola \\
\hline $\mathbf{1 8 7}$ & AKL-222 & NBPGR, Akola \\
\hline $\mathbf{1 8 8}$ & AKL-187 & NBPGR, Akola \\
\hline $\mathbf{1 8 9}$ & AKL-216 & NBPGR, Akola \\
\hline $\mathbf{1 9 0}$ & AKL-29 & NBPGR, Akola \\
\hline $\mathbf{1 9 1}$ & AKL-90 & NBPGR, Akola \\
\hline $\mathbf{1 9 2}$ & AKL-227 & NBPGR, Akola \\
\hline $\mathbf{1 9 3}$ & AKL-200 & NBPGR, Akola \\
\hline $\mathbf{1 9 4}$ & AKL-92 & NBPGR, Akola \\
\hline $\mathbf{1 9 5}$ & AKL-183 & NBPGR, Akola \\
\hline $\mathbf{1 9 6}$ & AKL-176 & NBPGR, Akola \\
\hline $\mathbf{1 9 7}$ & AKL-191 & NBPGR, Akola \\
\hline $\mathbf{1 9 8}$ & AKL-165 & NBPGR, Akola \\
\hline $\mathbf{1 9 9}$ & AKL-164 & NBPGR, Akola \\
\hline $\mathbf{2 0 0}$ & AKL-192 & NBPGR, Akola \\
\hline
\end{tabular}

Table.2: Meteorological data of experimental site for the year 2015

\begin{tabular}{|l|l|c|c|c|}
\hline Year & Months & Temperature $\left({ }^{\circ} \mathbf{C}\right)$ & Relative humidity (\%) & Rainfall (mm) \\
\hline $\mathbf{2 0 1 5}$ & January & 21.32 & 61.03 & 0.59 \\
\hline & February & 23.10 & 50.72 & Nil \\
\hline & March & 25.34 & 58.70 & $2 \mathrm{~mm}(25.03 .2015)$ \\
\hline & April & 25.87 & 66.55 & Nil \\
\hline
\end{tabular}

Discovering per cent contribution of traits to the total variability

Among the six variables studied, proline content (21.03) had highest per cent contribution to the total variability possessed by physiological traits followed by leaf water potential (18.63), spad chlorophyll meter reading (18.61), relative water content (16.82), harvest index (13.30) and specific leaf area (11.58) (table 8 and figure 1). Our results are on par with the results of Mohammad and Sharif (2015) who reported that factor analysis indicated four independent factors explaining $75 \%$ of total variability in control condition and $78 \%$ variability in drought stress condition in mung bean. Srikanth et al., (2017) has also reported similar findings on principal component analysis. 
Table.3 Summary of augmented ANOVA for grain yield and physiological traits of germplasm accessions under drought condition

\begin{tabular}{|l|c|c|c|c|c|c|c|c|}
\hline Sources of Variations & DF & HI & SCMR & LWP & PC & RWC & SLA & SYPP \\
\hline Blocks (b) & 4 & $247.54 * *$ & $396.55 * *$ & $1.17 * *$ & $470.90 * *$ & $423.68 *$ & $4067.34 *$ & $2.11 * *$ \\
\hline $\begin{array}{l}\text { Entries (e) } \\
\text { (Genotypes + Checks) }\end{array}$ & 204 & $54.41 *$ & $98.71 * *$ & $2.45 * *$ & $1707.90 * *$ & $425.40 * *$ & $4283.10 * *$ & $7.01 * *$ \\
\hline Checks & 4 & $64.39 *$ & 24.49 & $0.82 * *$ & $942.07 * *$ & 63.06 & 1924.20 & $3.76 * *$ \\
\hline Genotypes & 199 & $53.01 *$ & $79.58 *$ & $2.33 * *$ & $1712.67 * *$ & $433.68 * *$ & $4294.15 * *$ & $7.10 * *$ \\
\hline Checks vs Genotypes & 1 & $293.20 * *$ & $4203.25 * *$ & $32.57 * *$ & $3822.09 * *$ & 227.32 & $11518.68 * *$ & $0.42 *$ \\
\hline Error & 16 & 19.57 & 31.14 & 0.03 & 1.48 & 130.64 & 1339.95 & 0.09 \\
\hline
\end{tabular}

*Significant at $\mathrm{P}=0.05, * *$ Significant at $\mathrm{P}=0.01$

Table. 4 Correlation matrix (Pearson (n))

\begin{tabular}{|c|c|c|c|c|c|c|c|}
\hline Variables & HI & SCMR & LWP & PC & RWC & SLA & SYPP \\
\hline HI & 1 & $0.70^{*}$ & $0.70^{*}$ & $0.74^{*}$ & $0.61^{*}$ & $0.34^{*}$ & $0.60^{*}$ \\
\hline SCMR & $0.70^{*}$ & 1 & $0.80^{*}$ & $0.91^{*}$ & $0.79^{*}$ & $0.60^{*}$ & $0.62^{*}$ \\
\hline LWP & $0.70^{*}$ & $0.80^{*}$ & 1 & $0.93^{*}$ & $0.77^{*}$ & $0.60^{*}$ & $0.61^{*}$ \\
\hline PC & $0.74^{*}$ & $0.91^{*}$ & $0.93^{*}$ & 1 & $0.86^{*}$ & $0.67^{*}$ & $0.63^{*}$ \\
\hline RWC & $0.61^{*}$ & $0.79^{*}$ & $0.77^{*}$ & $0.86^{*}$ & 1 & $0.66^{*}$ & $0.51^{*}$ \\
\hline SLA & $0.34^{*}$ & $0.60^{*}$ & $0.60^{*}$ & $0.67^{*}$ & $0.66^{*}$ & 1 & $0.41^{*}$ \\
\hline SYPP & $0.60^{*}$ & $0.62^{*}$ & $0.61^{*}$ & $0.63^{*}$ & $0.51^{*}$ & $0.41^{*}$ & 1 \\
\hline
\end{tabular}

Values in bold* are significantly different at alpha $=0.05$

TP : Threshing \% ; RWC : Relative water content (\%); HI : Harvest index (\%); SLA : Specific leaf area; SCMR : SPAD Chlorophyll meter reading; SYPP : Seed yield plant-1

LWP : Leaf water potential(Mpa); PC : Proline content ( $\mu \mathrm{g} g-1)$

Table.5 Kaiser-Meyer-Olkin measure of sampling adequacy

\begin{tabular}{|l|l|l|l|l|l|l|}
\hline Traits & HI & SCMR & LWP & PC & RWC & SLA \\
\hline KMO Values & 0.94 & 0.90 & 0.82 & 0.81 & 0.93 & 0.94 \\
\hline
\end{tabular}

Table.6 Eigen values of principal component analysis

\begin{tabular}{|l|c|c|c|c|c|c|}
\hline \multicolumn{1}{|c|}{ Descriptives } & F1 & F2 & F3 & F4 & F5 & F6 \\
\hline Eigen value & 4.59 & 0.68 & 0.28 & 0.22 & 0.18 & 0.02 \\
\hline Variability (\%) & 76.60 & 11.43 & 4.69 & 3.78 & 3.10 & 0.38 \\
\hline Cumulative \% & 76.60 & 88.03 & 92.73 & 96.51 & 99.61 & 100.00 \\
\hline
\end{tabular}


Table.7 Factor loadings / component coefficient values of PCA

\begin{tabular}{|l|c|c|c|c|c|c|}
\hline \multicolumn{1}{|c|}{ Traits } & F1 & F2 & F3 & F4 & F5 & F6 \\
\hline Harvest index & 0.78 & -0.51 & -0.25 & -0.22 & -0.04 & -0.006 \\
\hline $\begin{array}{l}\text { Spad chlorophyll meter } \\
\text { reading }\end{array}$ & 0.92 & -0.07 & 0.05 & 0.07 & 0.35 & -0.04 \\
\hline Leaf water potential & 0.92 & -0.09 & 0.01 & 0.28 & -0.21 & -0.06 \\
\hline Proline content & 0.98 & -0.03 & 0.04 & 0.11 & -0.004 & 0.12 \\
\hline Relative water content & 0.87 & 0.16 & 0.34 & -0.26 & -0.08 & -0.01 \\
\hline Specific leaf area & 0.72 & 0.61 & -0.30 & -0.06 & -0.007 & -0.008 \\
\hline
\end{tabular}

Table.8 Per cent contribution of the physiological traits to the total variability in PCA

\begin{tabular}{|l|c|c|c|c|c|c|}
\hline \multicolumn{1}{|c|}{ Traits } & F1 & F2 & F3 & F4 & F5 & F6 \\
\hline Harvest index & 13.30 & 39.20 & 22.89 & 23.06 & 1.33 & 0.18 \\
\hline $\begin{array}{l}\text { Spad chlorophyll meter } \\
\text { reading }\end{array}$ & 18.61 & 0.81 & 1.13 & 2.75 & 68.46 & 8.22 \\
\hline Leaf water potential & 18.63 & 1.24 & 0.09 & 36.43 & 25.82 & 17.77 \\
\hline Proline content & 21.03 & 0.17 & 0.70 & 6.01 & 0.008 & 72.07 \\
\hline Relative water content & 16.82 & 4.13 & 43.23 & 30.01 & 4.32 & 1.45 \\
\hline Specific leaf area & 11.58 & 54.42 & 31.94 & 1.71 & 0.02 & 0.29 \\
\hline
\end{tabular}

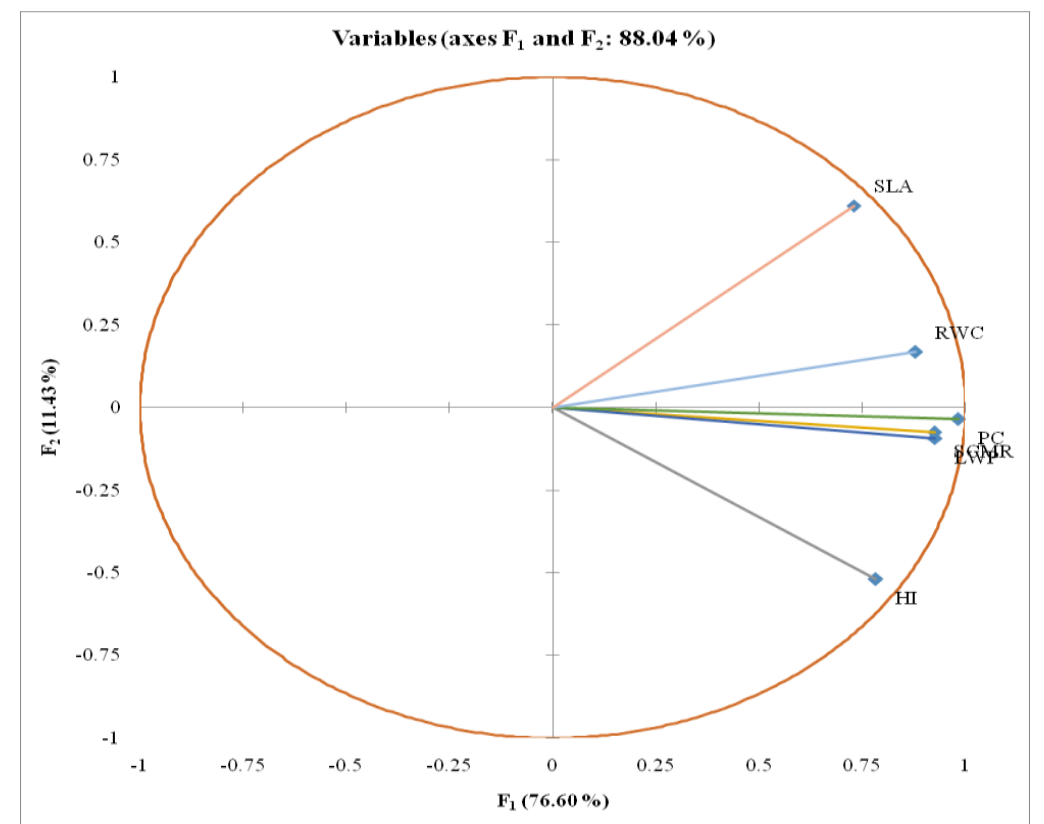

Figure.1 Loading plot of principal component analysis for six drought tolerant physiological variables 
Principal component analysis identified only two factors out of six with eigenvalues more than or close to one contributing for more than $88.03 \%$ of variability.Among the variables studied, variable proline content (11.91) had highest per cent contribution to the total variability followed by spad chlorophyll meter reading (10.80), leaf water potential (10.75), Relative water content (8.51) and specific leaf area (6.31) in green gram under drought condition.

\section{Acknowledgement}

Dr. Kanavi, M.S.P., thanks Director of Research, University of Agricultural Sciences, Bangalore for giving financial assistance to carry out the research work

\section{References}

Baroowa, B. and Gogoi, N. 2015. Changes in plant water status, biochemical attributes and seed quality of black gram and green gram genotypes under drought. Int. lett. Nat. Sci., 42,1-12.

Clarke, J.M. and Townley-Smith, T.F. 1984, Screening and selection techniques for improving drought tolerance. Crop Breeding: A Contemporary Basis. Pergamon Press, Oxford, UK, pp.,137162.

Dai, A. 2012. Increasing drought under global warming in observations and models. Nat. Clim. Chang.,3(52), 5258.

Federer, W.T. 1956. Augmented (or hoonuiaku) designs. The Hawaiian Planters' Record. LV (2),191-208.

Graham, P. H. and Vance, C. P. 2003.Legumes: importance and constraints to greater use.Plant Physiol., 131, 872-877.

Hemavathy, T. A., Shunmugavalli, N. and Anand, G. 2014. Genetic variability, correlation and path coefficient studies on yield and its components in mung bean (VignaradiataL. Wilczek). Indian J. Agric. Res.,38(4): 442-446.

Iqbal, Q., Saleem, M.Y., Hameed, A. and Asghar, M. 2014. Assessment of genetic diversity in tomato through agglomerative hierarchical clustering and principal component analysis. Pakistan J. Bot., 46(5), 1865-1870.

Kabita Mishra., S. Panigrahi D. Sarangi. 2018. Evaluation of cluster front line demonstration in greengram crop. International Journal of Current Microbiology and Applied Sciences., 7(10), 3344-3350.

Kursar, T.A., Engelbrecht, B.M., Burke, A., Tyree, M.T., Ei Omari, B. 2009, Tolerance to low leaf water status of tropical tree seedlings is related to drought performance and distribution. Funct. Ecol.,23: 93-102.

Maréchaux, I., Bartlett, M.K., Sack, L., Baraloto, C., and Engel, J. 2015, Drought as predicted by leaf water potential at turgor loss point varies strongly across species within an Amazonian forest. Functional Ecology.,29, 1268-1277.

Mohammad, Ghanbariand Sharif, Mollashahi, Javan. 2015. Study of the response of mung bean genotypes to drought stress by multivariate analysis. Int. J. Agric. Innov. Res., 3(4), 1298-1302.

Pooja, Bangar., Ashok, Chaudhury., Bhavana, Tiwari., Sanjay kumar., Ratnakumari. andKangila, Venkataramana, Bhat. 2019.Morphophysiological and biochemical response of mung bean [Vignaradiata(L.) Wilczek] varieties at different developmental stages under drought stress.Turkish $J$. Biol.,43(1), 58-69.

Rajeev Singh, R.,Singh, M.K.,Singh, A.K.,Singh, C., 2018. Pulses production in India: issues and elucidations. 
Innovation., 7(1), 10-13.

Reddy, A.A., 2009, Pulses production technology: status and way forward. Econ. Political Wkly., 44(52), 73-80.

Rowland, M. Kamanga., Ernest, Mbegaand Patrick, Ndakidemi., 2018, Drought mechanisms in plants: physiological responses associated with water deficit stress in Solanum lycopersicum.Adv. Crop Sci. Technol., 6(3), 1-8.

Sandhiya, V. and Saravanan, S. 2018.Genetic variability and correlation studies in green gram (Vigna radiata L. Wilczek). Electron. J. Plant Breed.,9(3), 1094-1099.

Shao, H.B., Chu, L.Y., Jaleel, C.A., Manivannan, P., Panneerselvam, R. and Shao, M.A. 2009. Understanding water deficit stress induced changes in the basic metabolism of higher plants biotechnologically and sustainably improving agriculture and the eco- environment in arid regions of the globe. Crit. Rev. Biotechnol.,29, 131151.

Srikanth, Thippani.,Eshwari, K.B. and Bhave, M.H.V. 2017. Principal component analysis for yield components in green gram accessions (Vigna radiata L.). Int. J. Pure App. Biosci., 5(4), 246253.

Supratima M, Basu., Venkategowda, Ramegowda., Anujkumar and Andy Pereira. 2016. Plant adaptation to drought stress. Faculty Rev.,1554, 111.

Yaqub, M., Mahmood, T., Akhtar, M., Iqbal, M. M. and Ali, S. 2010.Induction of mung bean [Vigna radiata (L.) Wilczek] as a grain legume in the annual rice-wheat double cropping system.Pakistan J. Bot.,42,3125-3135.

\section{How to cite this article:}

Kanavi. M. S. P., N. Nagesha, G. Somu, B. T. Krishnaprasad and Rangaiah. S. 2020. Principal Component Analysis of Physiological Traits Governing Drought Tolerance in Germplasm Accessions of Green Gram [Vigna radiata (L.)]. Int.J.Curr.Microbiol.App.Sci. 9(03): 29432956. doi: https://doi.org/10.20546/ijcmas.2020.903.338 